\title{
Editorial: Introduction to the 37th Annual Gallery of Fluid Motion (Seattle, Washington, USA, 2019)
}

\author{
Gwynn J. Elfring ${ }^{1}$ and Antonino Ferrante ${ }^{2}$ \\ ${ }^{1}$ Department of Mechanical Engineering, University of British Columbia, Vancouver, \\ British Columbia, Canada V6T 1Z4 \\ ${ }^{2}$ Department of Aeronautics and Astronautics, University of Washington, Seattle, Washington 98105, USA
}

(Received 18 August 2020; published 12 November 2020)

DOI: 10.1103/PhysRevFluids.5.110001

The 37th Annual Gallery of Fluid Motion (GFM) was held at the American Physical Society (APS) Division of Fluid Dynamics Annual Meeting in Seattle, Washington November 23-26, 2019. The meeting featured approximately 3300 technical abstracts with an additional 103 entries to the GFM for display during the meeting (66 video and 37 poster submissions). Submissions were received from 17 countries with approximately $50 \%$ of submissions coming from institutions outside the United States. The work submitted represents a broad range of fluid dynamical topics being explored in academia, industry, and national laboratories today.

To select winners of the 2019 GFM, two panels of judges (ten for videos and four for posters) were assembled from scholars with a diverse background in fluid mechanics. As in previous years, judges evaluated entries on the basis of the beauty and aesthetic value of the work, the scientific and technical merit of the described research, and the originality and clarity in communication.

The panel for the videos was formed by a total of ten judges and each video was ranked by four panelists. From these ratings, the seven top ranked videos were reviewed by all the judges and chosen as winners. Posters were judged in person, with panelists scoring each poster individually, with the five winning entries having the highest average score. As in previous years, the top three winners in each category were designated Milton van Dyke Award winners and received a monetary prize in addition to the certificates and copy of An Album of Fluid Motion given to all winners. The winning entries, as well as many of the other submissions, are visible online at https://gfm.aps.org. The 2019 Gallery of Fluid Motion winners are listed below.

\section{Milton van Dyke Award video winners}

(1) V0013 Impaled droplets: On the breakup of drops impacting singularities.

Guillaume Durey, Quentin Magdelaine, Mathias Kasiulis, Hoon Kwon, Julien Mazet, Pierre Chantelot, Anaïs Gauthier, Christophe Clanet, David Quéré

Université PSL; École Polytechnique

(2) V0040 Crystal Critters.

Samantha McBride, Henri-Louis Girard, Kripa Varanasi

Massachusetts Institute of Technology

(3) V0082 Blooming patterns in drying drops.

Paul Lilin, Philippe Bourrianne, Guillaume Sintès, Irmgard Bischofberger

MIT; Princeton 


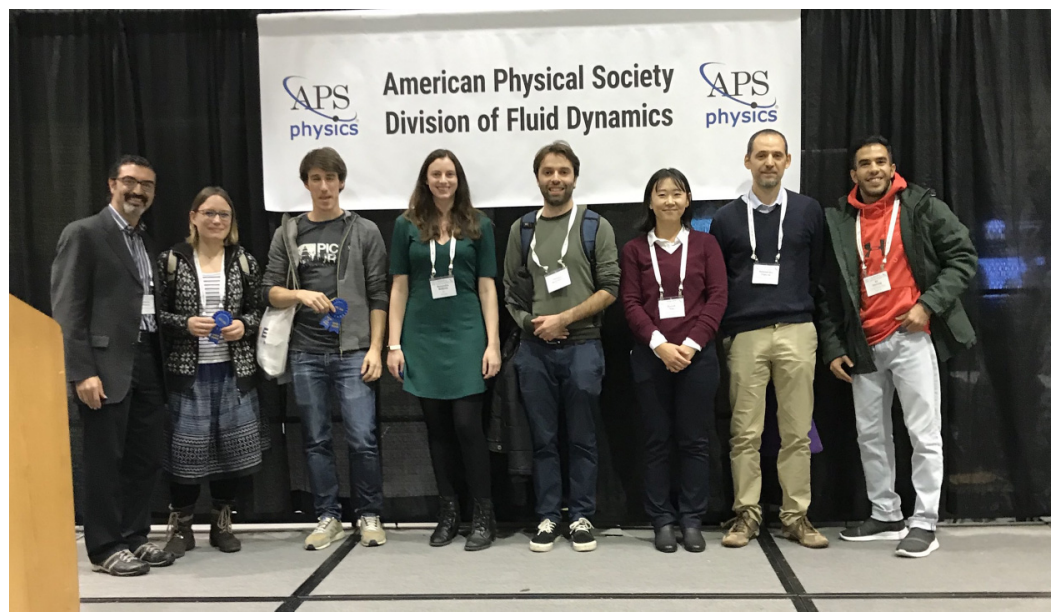

FIG. 1. Gallery of Fluid Motion video competition award winners (pictured with local GFM lead Antonino Ferrante).

\section{Gallery of Fluid Motion Award video winners}

(4) V0018 Breaking waves: to foam or not to foam?

Petr Karnakov, Sergey Litvinov, Jean M. Favre, Petros Koumoutsakos

ETH Zurich; Swiss National Supercomputing Centre

(5) V0036 Swinging jets.

Arnaud Bertsch, Alessandro Bongarzone, Eunok Yim, Philippe Renaud, François Gallaire École Polytechnique Fédérale de Lausanne

(6) V0067 Unraveling the interplay of two counter-rotating helical vortices.

Alessandro Capone, Francisco Alves Pereira

CNR-INM Institute of Marine Engineering

(7) V0072 Gas escape behavior from bursting bubbles.

Ali Al Dasouqi, David Murphy

University of South Florida

\section{Milton van Dyke Award poster winners}

(1) P0015 Gas giants' zonal jets in the laboratory.

Daphné Lemasquerier, Benjamin Favier, Michael Le Bars

Aix-Marseille University

(2) P0020 Rico and the jets.

Cristian Ricardo Constante Amores, Lyes Kahouadji, Assen Batchvarov, Omar K. Matar Imperial College London

(3) P0034 Fluid dynamics of Millefiori.

Cooper Kovar, Lisa Panczner, Heidi Reuter, Azar Eslam-Panah

Penn State University 


\section{Gallery of Fluid Motion Award poster winners}

(4) P0031 Hydrodynamic tweezing: Using water waves to push and pull. Ahmed Sherif, Leif Ristroph New York University

(5) P0035 Vortex bursting.

Wim M. van Rees

MIT

We would like to congratulate the winners in the 2019 edition of the Gallery of Fluid Motion and extend our sincere thanks to all the contributors for all the wonderful entries. We would also like to thank the 2019 Annual Meeting co-chairs, Alberto Aliseda and Dana Dabiri, for organizing such an outstanding conference and for entrusting in us the local organization of the GFM. Special thanks are due, as ever, to Kenneth Kiger, whose continued service as overall coordinator for the GFM ensures institutional memory and helps guide each local organizing committee in each iteration of the Gallery. We would also like to thank Peggy Holland and Margaret McDonald of Meetings and More for all their logistical support and finally thank Paul Dlug, Mark Doyle, and the staff of APS for their dedicated assistance and coordination throughout the GFM cycle. 